\title{
Anabases
}

ANABASES Traditions et réceptions de l'Antiquité

$14 \mid 2011$

Varia

\section{Gabriel DANZIG, Apologizing for Socrates: how Platon and Xenophon created our Socrates}

Jean-Marie Bertrand

\section{(2) OpenEdition}

Journals

Édition électronique

URL : http://journals.openedition.org/anabases/2365

DOI : 10.4000/anabases.2365

ISSN : 2256-9421

\section{Éditeur}

E.R.A.S.M.E.

\section{Édition imprimée}

Date de publication : 1 octobre 2011

Pagination : 256-258

ISSN : 1774-4296

\section{Référence électronique}

Jean-Marie Bertrand, «Gabriel DANzIG, Apologizing for Socrates: how Platon and Xenophon created our Socrates ", Anabases [En ligne], 14 | 2011, mis en ligne le 01 octobre 2011, consulté le 22 septembre 2020. URL : http://journals.openedition.org/anabases/2365 ; DOI : https://doi.org/10.4000/anabases. 2365

Ce document a été généré automatiquement le 22 septembre 2020

(c) Anabases 


\title{
Gabriel DANZIG, Apologizing for Socrates: how Platon and Xenophon created our Socrates
}

\author{
Jean-Marie Bertrand
}

\section{RÉFÉRENCE}

Gabriel DANZIG, Apologizing for Socrates : how Platon and Xenophon created our Socrates, Plymouth (UK), Lexington Books, 2010, $280 \mathrm{p}$. 44.95 livres-51.95 euros / ISBN 978-0-7391-3244-9.

1 Gabriel Danzig de l'Université Bar Ilan reprend dans ce volume, sous une forme révisée, trois articles parus récemment dans des revues bien connues et commodes d'accès : chapitre 1, "Plato and Xenophon on Socrates' Behavior in Court (The Apologies)", chapitre 2, «Building a Community under Fire (Crito)», chapitre 6, « Why Socrates Was Not a Farmer : Xenophon's Apology for Socrates (Oeconomicus) »; il y ajoute deux textes neufs : un chapitre 3, portant sur l'Euthyphron, «Disgracing Meletos", qui aurait pu faire lui aussi l'objet d'un article, un chapitre 5, «Plato's Socratic Seductions » sur le Lysis qui fait pendant à un texte qui constitue le chapitre 4, développé d'un article plus ancien traitant des Mémorables de Xénophon, "Xenophon's Socratic Seductions». Les deux indices, Names (il comprend curieusement, noms de personnes antiques, noms d'auteurs contemporains mentionnés dans les notes, titres d'ouvrages antiques) et Terms, sont tout à fait insuffisants. À la lecture de l'introduction qui veut donner sens à la construction de ce recueil, on apprend qu'il s'agit de comprendre que Platon aurait écrit ses premiers dialogues pour répondre à la campagne de diffamation qui s'en prenait à l'ensemble des disciples de Socrate après sa mort. Il ne faudrait donc pas s'intéresser aux développements philosophiques prétendus maladroits de ces textes mais s'en tenir à leur seule valeur apologétique. Xénophon joue une partition différente mais même l'Économique dont il est traité au chapitre 6 serait « a philosophic-apologetic 
work ». Du chapitre 1, on retiendra que Platon cherche à combattre l'impression que Socrate aurait été trop arrogant lors de son procès en le présentant comme simplement malhabile dans la vie sociale. Le second de ses discours d'audience et le troisième montrent combien il est humain dans ses doutes comme dans ses façons d'envisager des peines alternatives à la mort, dans l'évocation aussi de l'existence de ses fils en une sorte de péroraison qu'il prétend ne prononcer pourtant que parce qu'elle est désormais inutile pour obtenir la pitié. Cela donnerait à l'Apologie un ton conforme à ce que le tribunal attend d'un discours de défense, et donc le texte de Platon serait bien une plaidoirie acceptable par les Athéniens destinataires du texte. Il me semble néanmoins que ne devrait pas être négligé le fait que Platon en cette conclusion, par exemple, reconstruit l'image d'un Socrate imbu de lui-même puisqu'il s'assimile à un soldat mort pour la patrie en semblant demander à ses concitoyens de prendre en charge l'éducation de ses fils désormais orphelins; la revendication serait pour le moins malhabile si l'écriture n'était pas aussi maîtrisée. Xénophon pour sa part accorde à son personnage toutes les qualités qui en font un homme habile dans l'art de vivre en société et il sait s'y conduire en " maquereau ", étant master of the art of pimping; ainsi n'a-t-il été condamné à mort que parce qu'il l'avait expressément souhaité. Au chapitre 2, l'opposition établie entre les deux auteurs sur ce thème se réduit, le Platon du Criton rejoignant la position tenue par Xénophon dans sa propre Apologie. Le dialogue serait essentiellement destiné à ressouder la communauté des socratiques, les faiblesses d'argumentation doctrinale qu'on y relève n'auraient guère d'importance. Je me demande pourquoi le Phédon n'a pas fait l'objet d'une étude qui aurait infirmé ou non la validité de cette opinion. La " Prosopopée » est traitée, d'autre part, dans son rapport à la seule apologétique alors qu'elle est, à mon sens, un des fondements de la pensée politique platonicienne. Pour cette raison, on ne peut considérer le Criton comme un ouvrage de circonstance. Du chapitre 6, on retiendra l'idée que l'Économique a peu de chance de passer pour un prolongement de l'enseignement socratique mais que c'est un moyen pour Xénophon de prétendre, par fidélité à sa jeunesse, avoir pour le genre de vie qu'il avait choisi l'approbation de son maitre en proposant une sorte de second best. Dans les chapitres 4 et 5, on remarquera que le premier montre que, pour Xénophon, l' elenchos est un moyen utilisé par Socrate pour soumettre et s'attacher autrui après avoir détruit toute l'estime qu'il peut avoir de soi, que le second traitant essentiellement du Lysis insiste sur le fait que Socrate use de son intelligence pour se jouer des êtres jeunes et un peu bêtes, tout en restant indifférent à leur personne - je vois mal en quoi cela peut passer pour une défense du personnage. Le chapitre 3 portant sur l'Euthyphron est le seul qui soit vraiment une nouveauté dans ce recueil. L'idée essentielle est que ce discours doit être considéré comme une attaque contre Mélétos servant à la défense de Socrate. Une note signale que the dialogue serves other purposes as well mais que le problème philosophique is not the architectonic theme. Socrate est présenté dans le dialogue comme fondamentalement différent de celui qui est stigmatisé dans les Nuées par son refus de penser qu'un fils puisse penser devoir s'en prendre à son père. Euthyphron se voit comme un autre Socrate, mais l'aveu de cette prétention quelque peu grotesque permet de montrer par contraste tout ce que Socrate a de qualités intellectuelles et de vertu. Il est en même temps un second Mélètos puisqu'il s'en prend sans en avoir le droit à quelqu'un qu'il devrait respecter, le convaincre de son incapacité est bien plaider contre Mélètos. La conclusion du dialogue où reproche est fait à Euthyphron qui s'enfuit de ne pas vouloir enseigner son interlocuteur reprend l'idée que Mélètos est le véritable coupable de ce dont il accuse 
Socrate puisque lui non plus n'a voulu ni l'éduquer ni même le rencontrer comme on le sait de l'Apologie. L'ensemble est quelque peu paraphrastique et la conclusion est faible : Socrate est désireux d'apprendre ce qu'est la piété, in this he stood in stark contrast to his impious prosecutors. Je ne pense pas que ce genre d'argument pourrait convaincre un jury d'acquitter celui qui en use. Au plan de l'analyse littéraire, d'ailleurs, il semble que l'on néglige ce qu'est l'ironie de Socrate ou plutôt celle du cygne né sur ses genoux. Pour conclure, il me semble que l'on peut s'interroger sur la raison qui a conduit à la publication.

\section{AUTEURS}

\section{JEAN-MARIE BERTRAND}

Université Paris 1 Panthéon-Sorbonne

Jean-Marie.Bertrand@univ-paris1.fr 